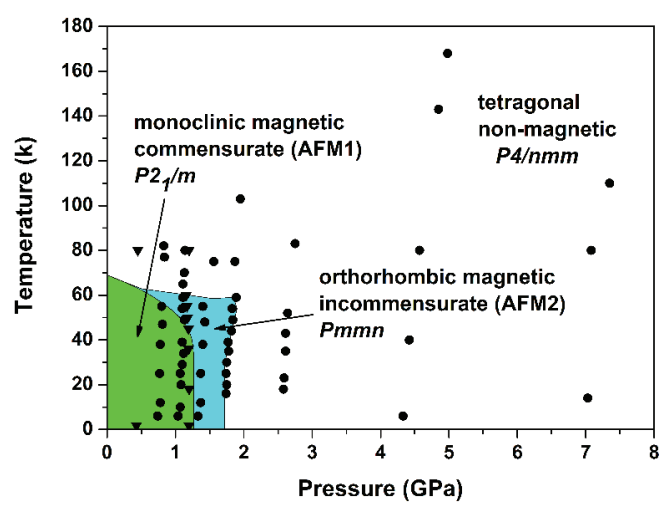

Figure 1. $p, T$-phase diagram of $\mathrm{Fe}_{1087}$ Te. The tetragonal $P 4 / \mathrm{nmm}$ phase is paramagnetic while the monoclinic $P 21 / \mathrm{m}$ and the orthorhombic Pmmn phases are magnetically ordered. Experimental data points are marked with - (this work) and $\boldsymbol{\nabla}$ (Ref. 2).

Keywords: Magnetic structure, high-pressure, neutron diffraction

\section{MS25-P6 Magnetic structures in complex materials revealed by resonant soft X-ray diffraction}

Eugen Weschke ${ }^{1}$, Schierle Enrico ${ }^{1}$, Frano Alex², Keimer Bernhard $^{2}$

1. Helmholtz-Zentrum für Materialien und Energie, Albert-Einstein-Str. 15, D-12489 Berlin, Germany

2. Max-Planck-Institut für Festkörperforschung, Heisenbergstrasse 1, D-70569 Stuttgart, Germany

email: eugen.weschke@helmholtz-berlin.de

With magnetic scattering factors as large as $200 \mathrm{r}_{0}$ (classical electron radius) [1], x-ray diffraction using resonant photon energies in the soft $\mathrm{x}$-ray regime provides particular sensitivity to magnetic ordering in transition metals by directly addressing the $3 \mathrm{~d}$ or $4 \mathrm{f}$ electronic states. The corresponding wavelengths are of the order of $1 \mathrm{~nm}$, yielding a rather small Ewald sphere. Notwithstanding, there are a number of important materials that can hardly be studied by other methods. From only one accessible magnetic Bragg peak detailed information about magnetic structures can be obtained, as discussed for a few examples.

Applied to a thin epitaxial film of the simple antiferromagnet EuTe, the method yields magnetic diffraction peaks that exhibit detailed Laue oscillations (Fig. 1). The temperature dependence of these profiles can be analyzed to obtain layer-resolved magnetization profiles [2].

Nickelate superlattices composed of layers of $\mathrm{LaNiO}_{3}$ (LNO) and $\mathrm{LaAlO}_{3}$ (LAO) or $\mathrm{DyScO}_{3}$ (DSO) can be grown with excellent structural quality with varying substrate-induced strain. While bulk LNO is nonmagnetic, dimensionality-driven magnetic order was observed in the superlattices for nickelate layers of 2 unit cells thickness. In addition, by exploiting the polarization dependence of resonant magnetic scattering, strain-dependent spin-moment directions could be determined, paving a way for controlling magnetic order [3].

For bulk crystals of multiferroic $\mathrm{DyMnO}_{3}$, the availability of circular polarized x-rays in combination with the magnetic sensitivity at resonance could be used to determine the chirality of the magnetic structures that are intimately linked to the ferroelectric properties of the material [4].

References

[1] J. Fink et al., Resonant elastic soft x-ray diffraction. Rep. Prog. Phys. 76, 056502 (2013).

[2] E. Schierle et al., Antiferromagnetic Order with Atomic Layer Resolution in EuTe(111) Films. Phys. Rev. Lett. 101, 267202 (2008).

[3] A. Frano et al., Orbital Control of Non-collinear Magnetic Order in Nickel Oxide Heterostructures. Phys. Rev. Lett. 111, 106804 (2013).

[4] E. Schierle et al., Cycloidal order of $4 f$ moments as a probe of chiral domains in $\mathrm{DyMnO}$. Phys. Rev. Lett. 105, 167207 (2010). 


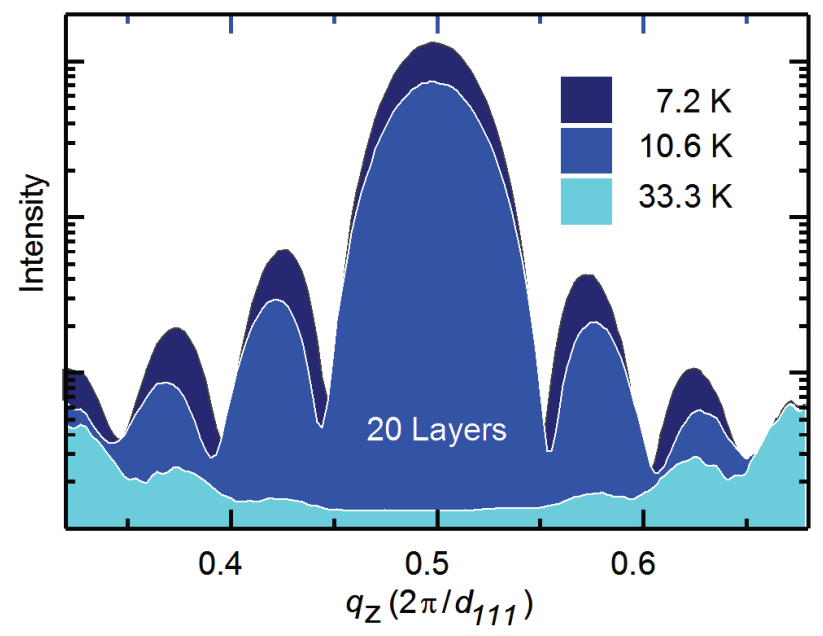

Figure 1. $\left(1 / 2 \frac{1}{1 / 2} 1 / 2\right)$ magnetic diffraction peak recorded from 20 layers of EuTe at the Eu-M $\mathrm{M}_{5}$ resonance. The temperature-dependent Laue oscillations ${ }^{5}$ can be analyzed to provide the layer-resolved magnetizations of the film.

Keywords: Resonant $\mathrm{x}$-ray scattering, transition metal oxides, thin films, heterostructures, multiferroics

\section{MS25-P7 Antiferromagnetic ordering in selected TmTX $(\mathrm{T}=$ transition metal; $\mathrm{X}-$ p-electron element) intermetallics}

Stanislaw Baran ${ }^{1}$, Andrzej Szytula ${ }^{1}$, Dariusz Kaczorowski ${ }^{2}$, Anthony Arulray ${ }^{3}$, Francoise Damay ${ }^{4}$, Andreas Hoser ${ }^{3}$, Boguslaw Penc $^{1}$

1. Marian Smoluchowski Institute of Physics, Jagiellonian University, ul. prof. Stanisława Łojasiewicza 11, PL-30-348 Kraków, Poland

2. Institute of Low Temperature and Structure Research, Polish Academy of Sciences, P. O. Box 1410, PL-50-950 Wroclaw, Poland

3. Helmholtz-Zentrum Berlin für Materialen und Energie $\mathrm{GmbH}$, Hahn-Meitner Platz 1, D-14-109 Berlin, Germany

4. Laboratoire Leon Brillouin, UMR 12, CEA-Saclay, CEA-CNRS, F-91191 Gif-sur-Yvette Cedex, France

email: stanislaw.baran@uj.edu.pl

Ternary intermetallics of general composition RTX (R - rare eatrh element; $\mathrm{T}=$ transition metal; $\mathrm{X}$ - p-electron element) are interesting group of compounds which draws researchers' interest for several decades. Among these compounds special concern is attracted to those crystallizing in the $\mathrm{ZrNiAl}$-type structure. This hexagonal structure (space group P-62m) consists of layers containing rare earth atoms separated by layers consisting of two remaining elements. Within one layer the rare earth atoms form a distorted kagome lattice. Such a triangular arrangement of atoms having localized magnetic moments may lead to magnetic frustration in case of antiferromagnetic interactions.

In this study we report on physical properties of six thulim intermetallics crystallizing in the ZrNiAl-type structure, namely: TmTIn ( $\mathrm{T}=\mathrm{Ni}, \mathrm{Pt}, \mathrm{Pd})$ and $\mathrm{TmAgX}$ $(\mathrm{X}=\mathrm{Si}, \mathrm{Ge}, \mathrm{Sn})$. The compounds have been investigated by means of magnetometric, heat capacity and electrical resistivity measurements. Their magnetic structures were derived from neutron diffraction data. All investigated compounds show antiferromagnetic ordering with Néel temperatures not exceeding $4.2 \mathrm{~K}$. Among magnetic structures both the commensurate ones as well as incommensurate with crystal structure are found. Especially interesting is the case of magnetic structure in $\mathrm{TmAgX}(\mathrm{X}=\mathrm{Si}, \mathrm{Ge})$ where to each of three magnetic moments, present in the crystal unit cell, a different propagation vector is applied. Magnetic structure determination has been supported by a symmetry analysis.

Keywords: magnetic structure, neutron diffraction, rare earth intermetallics 